\title{
Balanced Cluster Lifetime Prolonging Protocol for Wireless Sensor Network
}

\author{
Nidhi Rawat \\ M.Tech CSE \\ Uttarakhand Technical \\ University \\ Dehradun, India
}

\author{
Anshika Garg \\ Assistant Professor \\ Uttarakhand Technical \\ University \\ Dehradun, India
}

\author{
Pooja Khulbe \\ M.Tech CSE \\ Uttarakhand Technical \\ University \\ Dehradun, India
}

\begin{abstract}
A wireless sensor network with a large number of tiny sensor nodes can be used as an effective tool for gathering data in diverse conditions. One of the crucial matter in wireless sensor networks is developing an energy-efficient routing protocol which has a significant impact on the overall lifetime of the sensor network. In this paper, we propose a balanced cluster routing protocol called balance cluster lifetime prolonging protocol (BCLPP). In BCLPP, we divide the network into four static quadrants which have almost equal number of nodes, which reduce the overhead of dynamic clustering thus increase the lifetime of our network. Simulation-based evaluations are performed to compare the performance of BCLPP against Energy-Efficient Protocol with Static Clustering for Wireless Sensor Networks (EEPSC) Our experiment results show that BCLPP outperforms EEPSC in terms of network lifetime and power consumption minimization.
\end{abstract}

\section{Keywords}

Clustering technique, Energy efficiency, network lifetime and wireless sensor network.

\section{INTRODUCTION}

Wireless Sensor Networks (WSNs) have emerged as research areas with an Overwhelming effect on practical application developments. They permit fine grain observation of the ambient environment at an economical cost much lower than currently possible. In hostile environments where human participation may be too dangerous sensor networks may provide a robust service. Sensor networks are designed to transmit data from an array of sensor nodes to a data repository on a server in wireless sensor network each sensor node is a tiny equipment that can gather data from its nearby places, and carry out simple computing, and interact with other sensor nodes or with the base station (BS). Such networks have been realized due to recent advances in micro electromechanical systems and are expected to be widely used for applications such as environment monitoring, home security, and earthquake warning [1]. Despite the infinite scopes of wireless sensor networks, they are limited by the node battery lifetime. Once they are deployed, the network can keep operating while there is sufficient power in battery . this is to bi considered critical as it is almost impossible to replace the node battery once deployed over an inaccessible area. Such constraints combined with a typical deployment of large number of sensor nodes, have put in many difficulties to the design and management of sensor networks and necessitate energy-awareness at all layers of networking protocol stack [2], [3]. In this paper we assume a sensor network model, similar to those used in [4]-[6], with the following properties:

- All sensor nodes are still and alike with a limited stored energy.

- The nodes are supplied with potential to control power supply to vary their transmitted power.

- Location of nodes are unknown to the nodes in the network.

- Environment sensing by nodes are done at a fixed rate and always has data to send to the base station.

- Base station is not in between nodes and it has its fix place.

In this paper, we propose a balanced cluster routing protocol called balance cluster lifetime prolonging protocol (BCLPP).BCLPP, we divide the network into four static quadrants which have almost equal number of nodes, which reduce the overhead of dynamic clustering thus the lifetime of network is increased .Simulation-based evaluations are done to compare the performance of BCLPP against EnergyEfficient Protocol with Static Clustering for Wireless Sensor Networks (EEPSC) . Our experiment results show that BCLPP outperforms EEPSC in terms of network lifetime and power consumption minimization. In each cluster, BCLPP selects the sensor node with highest residual energy as the cluster-head $(\mathrm{CH})$; thus, not only there is always one $\mathrm{CH}$ for every cluster, and the burden of dynamic clustering is removed.( BCLPP) is an alteration of (EEPSC) protocol. The main difference between BCLPP and EEPSC are described below:

- $\quad$ BCLPP benefits a new idea of balancing the clusters by dividing the network into quadrants using temporary- $\mathrm{CHs}$ and utilizes a new setup and responsible node selection phase.

- $\quad$ BCLPP utilizes static clustering phenomenon, thus reduce the overhead of dynamic clustering.

\section{PROTOCOL ARCHITECTURE}

BCLPP is a self-organizing, static clustering technique that produce clusters only once during the set of network accomplishment. The functioning of( BCLPP) is divided up into several rounds, where each round contains a cluster formation phase, $\mathrm{CH}$ selection phase, data collection phase . We discuss this phases in detail as follows. 


\subsection{Setup Phase}

BCLPP, firstly arrange the $\mathrm{Xi}$ and $\mathrm{Yi}$ coordinate of nodes in ascending order and short $\mathrm{Xi}$ and $\mathrm{Yi}$ to get half number of nodes and plot them on $\mathrm{X}, \mathrm{Y}$ axis and form these axis as a center coordinate. Afterward, the nodes greater than both coordinate $(\mathrm{Xi}, \mathrm{Yi})$ are put in one cluster. The nodes smaller than both coordinate $(\mathrm{Xi}, \mathrm{Yi})$ are put in one cluster. The nodes smaller than ( $\mathrm{Xi}$ ) but greater than (Yi) are put in one cluster. And the nodes smaller than (Yi) and greater than $\mathrm{Xi}$ are put in one cluster. Now all clusters have same number of nodes. The set-up phase is finish and the next phase can begin.

\subsection{Responsible Node Selection Phase}

After the clusters are recognized, network initiates its normal function and responsible nodes (temporary- $\mathrm{CH}$ and $\mathrm{CH}$ ) selection phase starts. At the starting of each round, every node sends its residual energy level to the temporary- $\mathrm{CH}$ in its time period. Later on, temporary- $\mathrm{CH}$ selects the sensor node with utmost energy level as $\mathrm{CH}$ for exist round to gather the data of sensor nodes of that cluster, perform local data aggregation, and converse with the sink; and the node with lowest energy level as temporary-CH for next round and sends a round-start packet including the new responsible sensor IDs for the current round. This packet also represents the starting of round to other sensor nodes. While every sensor node has a Pr-determined time period, changing the $\mathrm{CHs}$ has no effect on the schedule of the cluster functioning.

\subsection{STEADY STATE PHASE}

The steady-state phase is divided into frames where nodes send their data to the $\mathrm{CH}$ during per-determined time periods. These data consists of a node ID and the measure of parameters that are sensed. In the next section we see that the whole energy exhausted in the system is larger using multihop routing than direct communication to the base station and that's why, we use direct communication technique between $\mathrm{CH}$ and sink.

The duration of each slot in which data is broadcasted by node is persistent, and so the frame of data send depends on the number of nodes in the cluster. To reduce energy dissipation, the radio of each non-cluster head node is turned off until its allocated communication time, but the Cluster head should bi in state to receive all the data from nodes in the cluster.

\section{SIMULATION RESULTS}

\subsection{Simulation Environment}

\begin{tabular}{|c|c|}
\hline Parameter & Parameter's Value \\
\hline Network Area & $100 \mathrm{~m} \times 100 \mathrm{~m}$ \\
\hline Base Station's Position & $(50 \mathrm{~m}, 175 \mathrm{~m})$ \\
\hline Initial Energy for nodes & 2 Joule \\
\hline Number of deployed nodes & 100 \\
\hline Size of data message & $4000 \mathrm{bits}$ \\
\hline$E_{D A}$ & $5 \mathrm{~nJ}$ \\
\hline$E_{e l e c}$ & $50 \mathrm{~nJ}$ \\
\hline$\varepsilon_{t s}$ & $10 \mathrm{pJ} / \mathrm{bit} / \mathrm{m}^{2}$ \\
\hline$\varepsilon_{m p}$ & $0.0013 \mathrm{pJ} / \mathrm{bit} / \mathrm{m}^{4}$ \\
\hline
\end{tabular}

\subsection{Network Lifetime}

\subsubsection{Comparison between BCLPP and EEPSC}

The performance of BCLPP against EEPSC is carried out by a set of experiments

- At the end of network operation:

- Number of nodes alive in the network in BCLPP > Number of nodes alive in the network in EEPSC

- In BCLPP occurrence of first node's death happens after 400sec. but the same event in EEPSC occurs after $310 \mathrm{sec}$.

- $40.23 \%$ gain of BCLPP over EEPSC in terms of number of nodes alive in the network.

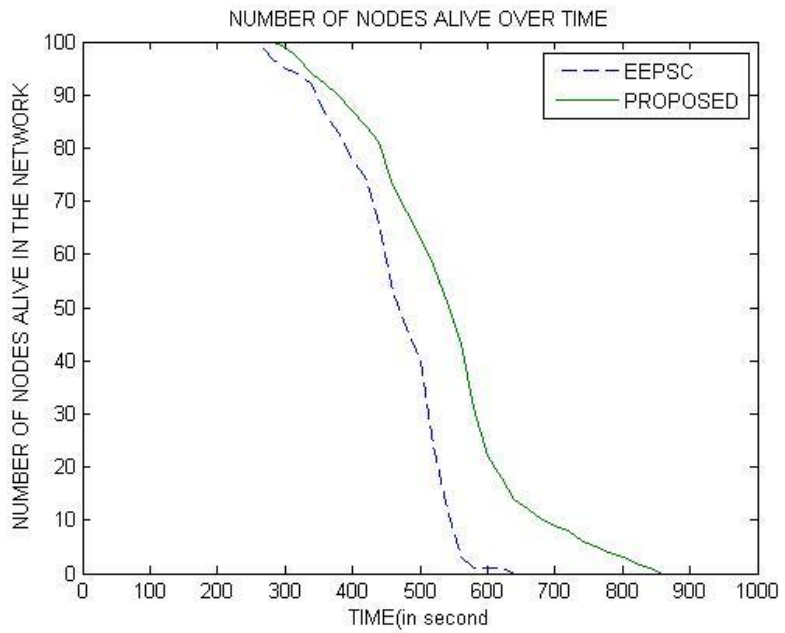

Fig 1. Number of nodes alive over time

\subsubsection{Average Energy per Node}

- The performance of BCLPP against EEPSC is carried out by a set of experiments

- At the end of network operation,

- Average Energy per node(in Joule) in BCLPP > Average Energy per node(in Joule) in EEPSC

- In BCLPP energy consumption is lower than that in EEPSC. Leading to long lasting energy hence enhancement in average energy per node.

- $\quad 45.45 \%$ gain of BCLPP over EEPSC in terms of number of nodes alive in the network. 


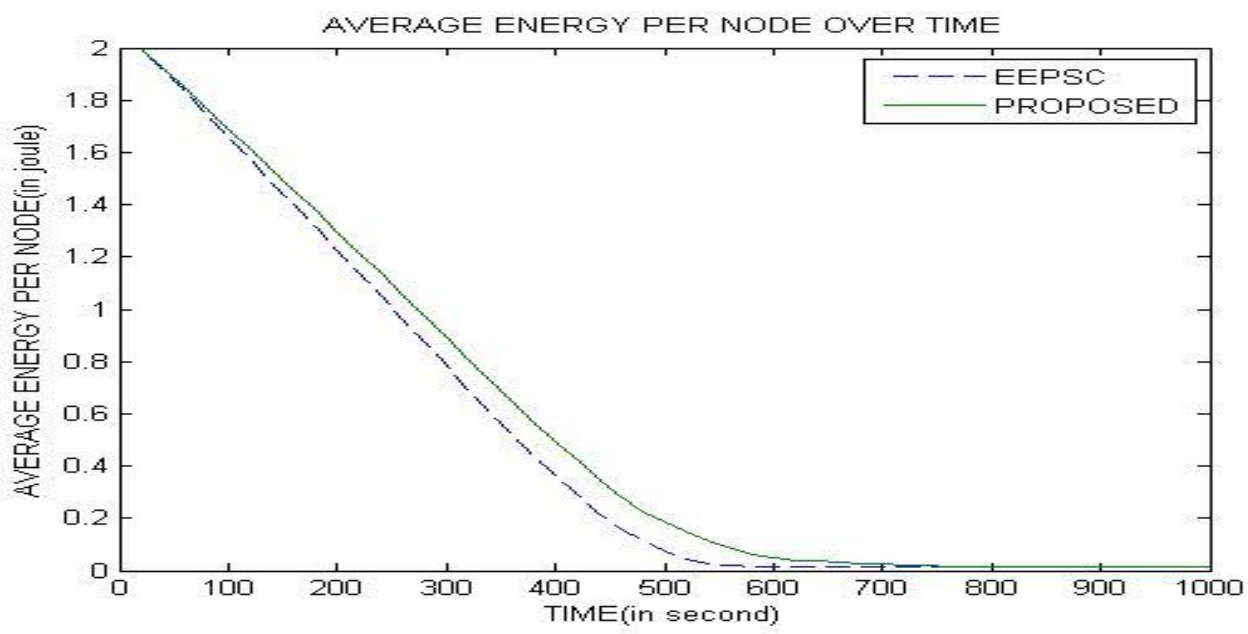

Fig 2. Average Energy per node over time

\section{CONCLUSION}

With the results of simulation, we can conclude that in the case of (EEPSC), the sensor nodes died earlier as they lose their energy while giving data to the $\mathrm{CH}$. This reduced the network life time and even the energy per node was decreased. And in case of EEPSC the clusters were not balanced as nodes in cluster were not equal. Energy dissipated in the node to cluster head was high in EEPSC. As the simulation time increases, nodes in the network continuously lose its energy and after a fix simulation time network collapse. And from the result we can see that in the proposed algorithm the numbers of nodes in cluster were almost equal and due to this the energy dissipated was less and node died less in comparison to that of EEPSC.

- $40.23 \%$ gain of BCLPP over EEPSC in terms of number of nodes alive in the network

- $45.45 \%$ gain of BCLPP over EEPSC in terms of number of nodes alive in the network.

\section{FUTURE SCOPE}

Work can be done on messages send to base station over time and energy.

- Cluster head comprises of one parameter only so we can use more parameters to form cluster head.

- We can further improve network lifetime and average energy.

- To investigate the proposed scheme with heterogeneous energy model of sensor nodes.

\section{REFERENCES}

[1] F. Zhao and L. Guibas, "Wireless Sensor Networks: An Information Processing Approach (Morgan Kaufmann Series in Networking)." San Mateo, CA: Morgan Kaufmann, 2004.

[2] Ding, W. and Marchionini, G. 1997 A Study on Video Browsing Strategies. Technical Report. University of Maryland at College Park.

[3] Fröhlich, B. and Plate, J. 2000. The cubic mouse: a new device for three-dimensional input. In Proceedings of the SIGCHI Conference on Human Factors in Computing Systems
[4] Tavel, P. 2007 Modeling and Simulation Design. AK Peters Ltd.

[5] Sannella, M. J. 1994 Constraint Satisfaction and Debugging for Interactive User Interfaces. Doctoral Thesis. UMI Order Number: UMI Order No. GAX9509398., University of Washington.

[6] Forman, G. 2003. An extensive empirical study of feature selection metrics for text classification. J. Mach. Learn. Res. 3 (Mar. 2003), 1289-1305.

[7] Brown, L. D., Hua, H., and Gao, C. 2003. A widget framework for augmented interaction in SCAPE.

[8] Akyildiz, W. Su, Y. Sankarasubramaniam, and E. Cayirci, "A survey on sensor networks", IEEE Communication Magazine, Aug. 2002.

[9] Wei Yen, Ching-Wei Chen and Cheng-hsiang Yang, "Single Gossiping with Directional Flooding Routing Protocol in Wireless Sensor Networks", in Proceedings IEEE, 2008.

[10] K. Sohrabi et al., "Protocols for self-organization of a wireless sensor network", IEEE Personal Communications 7 (5) (2000) 16-27.1990.

[11] Chalermek Intanagonwiwat, Ramesh Govindan, Deborah Estrin, John Heidemann, and Fabio Silva, "Directed Diffusion for Wireless Sensor Networking", in Proceedings IEEE/ACM, Vol. 11, No. 1, $\mathrm{Fb} 2003$.

[12] ] A. Howard, M. J. Mataric, and G. S. Sukhatme. "Mobile Sensor Network Deployment using Potential Fields: A Distributed, Scalable Solution to the Area Coverage Problem. In DARS 02, Fukuoka, Japan, June 2002.

[13] Brain P. Crow, Indra Widjaja, Jeon Geun Kim and Prescott T. Sakai, "IEEE 802.11 Wireless Local Area Networks", IEEE Communication Magazine, Vol.35, Sep 1997.

[14] Shio kumar ,singh,MP singh, and DK singh. "A Survey of Energy-Efficient Hierarchical Cluster-Based Routing in Wireless SensorNetworks" Int. J. of Advanced Networking and Applications), VOL: 02, Page: 570-580, (2010). 